\title{
Dynamic imaging of cancer invasion and metastasis: principles and preclinical applications
}

\author{
Peter Friedl
}

Published online: 20 February 2009

(c) The Author(s) 2009. This article is published with open access at Springerlink.com

Basic and applied cancer research are critically dependent upon methods to detect the size together with molecular and functional parameters of primary tumor lesions and metastases. For more than 150 years, the cellular basis of cancer growth and progression was predominantly studied using histopathological analysis of cells and tissues after fixation and staining. Only over the past decade, the advent of long-term 3D culture techniques, innovative live-cell multi-dimensional microscopy, and fluorescent reporter strategies have caused a middle-sized revolution in cancer research providing access to cell structure and function with cellular and subcellular resolution to living cells and molecules in 3D tissue environments in vitro and in vivo. Consequently, dynamic imaging has provided novel and often surprising insight into the hallmarks of cancer progression and metastatic dissemination. All authors articles included in this special issue of Clinical and Experimental Metastasis have substantially contributed to this progress. Old and often complicated questions begin to be elucidated, literally. How do cancer cells invade tissue? How do they coordinate their cytoskeleton with intracellular and extracellular proteases and adhesion cascades to generate a

P. Friedl $(\bowtie)$

Microscopical Imaging Centre, Department of Cell Biology, Nijmegen Center for Molecular Life Science, Radboud University Nijmegen Medical Centre, Geert Grooteplein 28, P.O. Box 9101, 6500 HB Nijmegen, The Netherlands e-mail: P.Friedl@ncmls.ru.nl

\section{P. Friedl}

Rudolf-Virchow Center for Experimental Biomedicine and Department of Dermatology, Venerology, and Allergology, University of Würzburg, Josef-Schneider-Strasse 2, 97080 Würzburg, Germany particular morphology and overcome tissue barriers? How do cancer cells coopt the tumor microenvironment and mislead the immune defense to maintain tolerance rather than effective cytotoxic killing? How do metastatic seeding and cross-talk with the stroma of secondary sites lead to macroscopic outgrowth of life-threatening metastasis? Lastly and importantly, how can we translate this knowledge to identify and validate targeted single- and multimodality therapy and combat cancer disease? The articles included in this special issue of CEM cover the spectrum from subcellular to whole-body imaging and discuss key aspects of cancer imaging in basic reseach and preclinical drug discovery.

The most basic basic process underlying cancer invasion and metastasis is a dynamic actomyosin cytoskeleton that, in response to external factors, drives cell polarity, turn over of cell matrix interactions and migration of cancer cells away from the primary lesion into surrounding tissues and blood and lymph vasculature. Olson and Sahai [1] review intracellular regulators of the actin cytoskeleton in cancer using modern 3D in vitro culture and intravital models monitored by sensitive microscopy. Whereas their specific role in the invasion cascade remains to be determined, it appears likely that at least some actin regulators drive particular steps of metastatic dissemination and cellular adaptation responses and, therefore, could be amenable for therapeutic targeting.

Besides mechanical coupling of the cell to its environment, the actin cytoskeleton also provides a scaffold that coordinates adhesion and proteolytic events at the cell surface. Whereas most studies address the location and function of surface proteases in 2D models which probably are inappropriate to capture cancer cell invasion into the tissue stroma, Wolf and Friedl [2] show how 3D multimodal confocal microscopy allows to identify location, 
function and structural consequences of collagenase activity in cancer cell migration. These studies provide a subcellular-resolved map of quite different actin-rich proteolytic cell structures that execute tissue remodeling during invasion.

Cancer cell-derived proteases including matrix metalloproteinases, serine proteases and cathepsins further execute important functions in protein processing and cellcell communication. Sameni et al. [3] present a live-cell coculture model to dyamically monitor where and how different protease classes become upregulated and activated at the interface between cancer cells, extracellular matrix, and reactive stroma cells. Because in many cancer types upregulated proteases are strongly associated with cancer progression and poor outcome, such methodology may be useful for screening up- and downsstream regulators of the tumor-stroma interaction and the identification of drugs that may inhibit this tumor-promoting process.

As concequence of a deregulated tumor-stroma margin, the normal tissue structure becomes replaced by a reactive de-novo tissue comprising activated stromal cells, not dissimilar to chronic non-healing wounds. Advanced spectrally resolved multiphoton microscopy and the detection of the fluorescence life-time can decipher an emission "signature" of both, tumor, tumor-stroma and tumor-associated cells in life tissues. Highlighting fluorescence lifetime microscopy, Provenzano et al. [4] review the technology of multiphoton imaging and provide examples of disorganized tumor stroma from mouse models of cancer that may be instrumental in future diagnostic and therapeutic monitoring of preclincal and clinical samples.

Concommitant to cancer progression, many cancer types show an increased infiltration of activated cytotoxic $\mathrm{T}$ lymphocates that recognize tumor-associated antigens but fail to eliminate the disease. Mempel and Bauer [5] summarize dynamic experimental models and imaging techniques that allow to dynamically visualize immune-cell activation and killing of cancer cells. Besides providing better insight into immune escape strategies of cancer lesions, these approaches will also help to identify mechanisms of cell delivery and pharmacological interference to improve both, endogenous anti-tumor immune responses and adoptive cell delivery in cancer immunotherapy.

A traditional approach to visualize kinetic events in vascular biology in small animal models is the dorsal skin-fold chamber, which also provides direct optical access to cancer growth and regression and the reactive tumor stroma. Koehl and Geissler [6] review the technique of the skin-fold chamber, discuss stengths and pitfalls of the approach, and provide compelling examples for the use of dynamic imaging of the tumor vasculature and cancer growth in preclinical drug discovery. Anti- angiogenic therapy using inhibition of the mTor pathway has served as role model for a wealth of subsequent preclinical studies to detect principles and inhibition regimens of cancer progression.

Besides advances in microscopy, the introduction of fluorescent tumor models has revolutionized long-term monitoring of cancer progression and regression in small animal models. Robert Hoffman has pioneered the use of single- and dual-color tumor models in, often, fluorescent mouse models for non-invasive and invasive optical imaging. These studies have provided insight into mechanisms of cancer progression and regression. Here, he reviews the instrumentation allowing fluorescent imaging of different steps of cancer progression and metastasis in live animals, its use in different orthotopic models, and preclinical therapy of metastatic cancer progression and relapse [7].

After distant seeding, the growth of metastases is the main cause for lethality. Therefore, an understanding of organ-preferential seeding and growth is most relevant for targeting progressive cancer disease. Non-invasive multimodality imaging of live tumors in small animal models, such as combining computer tomography with bioluminescence imaging, has strongly enhanced our understanding of the mechanisms driving fatal outcome. Kaitzel and collegues [8] review current approaches of multimodality imaging in basic research and drug discovery, exemplified by bone metastasis and its treatment with bisphophonates. These imaging approaches are further instrumental in developing novel probes to monitor and target human cancer disease.

Modern live-cell imaging technology has not only boosted academic cancer research but also is being implemented into the drug discovery pipeline of the pharmaceutical industry. Neil O. Gallagher features the combination and sequence of approaches used for largescale screening and discovery of new compounds to target cancer invasion and metastatic progression [9]. Besides an impressive complementary infrastructure to monitor different cell functions in 3D models, image postprocessing, quantification and bioinformatic integration are key elements of modern drug discovery. Such technology will further help to define multi-targeted therapy using validated 3D models with in vivo relevance.

In summary, academic and applied cancer research greatly depend upon 3D cell culture techniques and imaging approaches which progressively complement and, in part, replace traditional strategies. I hope this special issue will be an eye-opening, fascinating introduction to a better-resolved and dynamic understanding of cancer disease during progression and, hopefully, therapy-induced regression. 
Open Access This article is distributed under the terms of the Creative Commons Attribution Noncommercial License which permits any noncommercial use, distribution, and reproduction in any medium, provided the original author(s) and source are credited.

\section{References}

1. Olsen MF, Sahai E (2009) The actin cytoskeleton in cancer cell motility. Clin Exp Metast. doi:10.1007/s10585-008-9174-2

2. Wolf K, Friedl P (2009) Mapping proteolytic cancer cellextracellular matrix interfaces. Clin Exp Metast. doi:10.1007/ s10585-008-9190-2

3. Sameni M, Cavallo-Medved D, Dosescu J, Jedeszko C, Moin K, Mullins SR, Olive MB, Rudy D, Sloane BF (2009) Imaging and quantifying the dynamics of tumor-associated proteolysis. Clin Exp Metast. doi:10.1007/s10585-008-9218-7

4. Provenzano PP, Eliceiri KW, Keely PJ (2009) Multiphoton microscopy and fluorescence lifetime imaging microscopy (FLIM) to monitor metastasis and the tumor microenvironment. Clin Exp Metast. doi:10.1007/s10585-008-9204-0

5. Mempel TR, Bauer CA (2009) Intravital imaging of $\mathrm{CD}^{+} \mathrm{T}$ cell function in cancer. Clin Exp Metast. doi:10.1007/s10585-0089196-9

6. Koehl GE, Gaumann A, Geissler EK (2009) Intravital microscopy of tumor angiogenesis and regression in the dorsal skin fold chamber: mechanistic insights and preclinical testing of therapeutic strategies. Clin Exp Metast. doi:10.1007/s10585-008-9234-7

7. Hoffman RM (2009) Imaging cancer dynamics in vivo at the tumor and cellular level with fluorescent proteins. Clin Exp Metast. doi:10.1007/s10585-008-9205-z

8. Kaijzel EL, Snoeks TJA, Buijs JT, van der Pluijm G, Lowik CWGM (2009) Multimodal imaging and treatment of bone metastasis. Clin Exp Metast. doi:10.1007/s10585-008-9217-8

9. Carragher NO (2009) Profiling distinct mechanisms of tumour invasion for drug discovery: imaging adhesion, signalling and matrix turnover. Clin Exp Metast. doi:10.1007/s10585-008-9222-y 